\title{
Sex Hormone Binding Globulin as a Predictor of in Vitro Fertilization Outcomes in Polycystic Ovary Syndrome: Our Results
}

\author{
Gaddas Meriem ${ }^{1 *}$, Chaouache Nesrine ${ }^{1}$, Ajina Mounir ${ }^{2}$, Tabka Zouhair ${ }^{1}$, \\ Zaouali Ajina Monia ${ }^{1}$ \\ ${ }^{1}$ Laboratory of Physiology and Functional Explorations, Faculty of Medicine "Ibn el Jazzar", \\ University of Sousse, Tunisia \\ 2Unity of Reproduction Medicine, "Farhat Hached" University Hospital, Sousse, Tunisia \\ Email: "meriem-gaddas@hotmail.fr
}

Received 14 May 2016; accepted 10 June 2016; published 13 June 2016

Copyright (C) 2016 by authors and Scientific Research Publishing Inc.

This work is licensed under the Creative Commons Attribution International License (CC BY). http://creativecommons.org/licenses/by/4.0/

(c) (i) Open Access

\section{Abstract}

Objectives: in case of Polycystic Ovary Syndrome (SOPK), predicting ovarian response before undertaking risky in vitro fertilization (IVF) proceeds is crucial. Sex Hormone Binding Globulin (SHBG) is introduced in literature as an interesting indicator of follicle's maturation and quality. The aim of this study was to investigate whether serum SHBG levels are related to fertilization outcomes in SOPK women undergoing IVF procedures. Methods: in a prospective cohort study, we enrolled 61 SOPK women and 91 matched group of infertile women. All of them were undergoing FIV/ICSI proceeds. SHBG levels were performed in peripheral blood samples as well as others hormones determination. Results: we noted significant differences between our study group of SOPK patients and the Control group concerning the characteristics of the ovarian response to gonadotrophins administration and concerning the profile of biological proceeds' outcomes. But no significant correlation between SHBG and FIV/ICSI outcomes was found either in SOPK or in Control group. Conclusions: our study provides evidence that SHBG can't be considered as a predictor of IVF outcomes because production of this steroid-binding protein depends on many parameters such as metabolic disorders frequently associated with SOPK.

\section{Keywords}

SOPK, FIV, SHBG

\footnotetext{
${ }^{*}$ Corresponding author.
}

How to cite this paper: Meriem, G., Nesrine, C., Mounir, A., Zouhair, T. and Monia, Z.A. (2016) Sex Hormone Binding Globulin as a Predictor of in Vitro Fertilization Outcomes in Polycystic Ovary Syndrome: Our Results. Open Journal of Obstetrics and Gynecology, 6, 404-410. http://dx.doi.org/10.4236/ojog.2016.67053 


\section{Introduction}

Concerning in vitro fertilization (IVF), follicular environment and fluid content are major determinants of follicle growth, oocyte maturation and could influence fertilization outcomes [1]. Sex Hormone-Binding Globulin (SHBG), primarily produced by the liver, is known to be the most important determinant of the biologically available steroids in human plasma [2]. Recently, mRNA encoding SHBG has been shown to be expressed in the ovaries and corpora lutea, as well as in luteinised granulosa cells from IVF follicular samples [1] [3] [4], which indicate a role of SHBG in local regulation of ovarian functions [5]. Furthermore, it has been suggested that SHBG may modulate the patient's response to gonadotropin treatment by controlling estradiol levels [5] [6]. The aim of this study was to investigate whether serum SHBG levels were related to fertilization outcomes in women undergoing IVF procedures. Specifically, we examined whether the correlation SHBG/ FIV outcomes differed from Polycystic Ovary Syndrome group (SOPK) to Control group of infertile women.

\section{Subjects and Methods}

\subsection{Study Population}

We enrolled a total of 152 infertile women who were devised into two groups: SOPK group and Control group, composed respectively by 61 and 91 patients.

The diagnosis of PCOS was based on the Rotterdam 2003 criteria that include the presence of two among three of the following features: clinical and/or biochemical hyperandrogenism, chronic anovulation and/or oligoamenorrhea and evidence of micropolycystic ovaries in ultrasonographic. We excluded women with other related disorders of hyperandrogenism such as congenital adrenal hyperplasia, hyperprolactinemia, or Cushing's syndrome.

The control group consisted of women with tubal and/or male-factor infertility, normal menstrual cycles (28 30 days) and no signs of hyperandrogenism.

Patient's information is documented in details, including age, weight, menstruation history, pelvic ultrasound examination results and basal hormone levels.

All patients underwent a controlled ovarian stimulation for in vitro Fertilization (IVF) or intracytoplasmic sperm injection (ICSI) based on a long GnRH agonist stimulation protocol. Specifically, 20IU of a GnRHagonist was administered daily starting in the mid luteal phase of the cycle. Administration of recombinant follicle stimulating hormone ( $\mathrm{rFSH}$, Gonal-F, Merk-Serono, Geneva, Switzerland) was started after at least 14 days of GnRH therapy and_when serum estradiol (E2) was less than $100 \mathrm{pmol} / \mathrm{l}$ and the thickness of the endometrium less than $5 \mathrm{~mm}$. The starting dose of rFSH was $150 \mathrm{IU}$ and was adjusted individually from day 6 of the cycle according to E2 values and ultrasonographic follicle measurements. An ovulatory dose of human chorionic gonadotropin $10000 \mathrm{IU}$ was administered when mean diameter of at least three follicles was larger than $16 \mathrm{~mm}$ in diameter and the plasma E2 concentration was higher than $1500 \mathrm{pmol} / 1$. Follicle aspiration for oocyte retrieval was performed $36 \mathrm{~h}$ after $\mathrm{hCG}$ administration.

Oocytes, with a diameter higher than $18 \mathrm{~mm}$, were retrieved by follicle aspiration by the transvaginal route under ultrasound guidance.

The details of the protocol were explained to the patients who gave their consent and the Hospital Ethics Committee approved the protocol.

\subsection{Hormonal Assays}

On the day of oocyte retrieval peripheral blood was collected in order to measure serum's levels of SHBG, E2, total testosterone (T) and $\Delta-4$ androstenedione $(\Delta-4)$.

Blood samples were centrifuged immediately after collection and equal aliquots of plasma were pooled and stored at $-20^{\circ} \mathrm{C}$ until assayed. Plasma E2, T and SHBG levels were determined by RIA (Radio Immuno Assay, Beckman Coulter, Czech Republic). The inter and intra coefficient of variation for SHBG, T, $\Delta-4$ and E2 were respectively $(\leq 8.6 \%, 6.1 \%),(\leq 11.2 \%, \leq 12.1 \%),(\leq 9.8 \%, \leq 5.6 \%)$ and $(\leq 15 \%, 14.8 \%)$.

Samples were assayed in duplicate. The free androgen index (FAI) was calculated using the following formula:

$$
[\mathrm{T}(\mathrm{nmol} / \mathrm{L}) \times 100] / \mathrm{SHBG}(\mathrm{nmol} / \mathrm{L})
$$




\subsection{Statistical Analysis}

Statistical significance was determined by Student's paired or unpaired $t$-test. Correlations were performed by Pearson's method. A P value of less than 0.05 was considered statistically significant. Results are expressed as the mean \pm standard error of the mean (SEM).

\section{Results}

\subsection{Clinical Characteristics}

Clinical characteristics of the study population and the control group were presented in Table 1. We found significant differences in the BMI and serum SHBG, Testosterone and $\Delta-4$ androstenedione levels between the two groups using the t-test.

No significant differences concerning patient's age, the FAI value or the E2 level on the hCG administration day were found.

\subsection{Characteristics of FIV/ICSI Outcomes}

The characteristics of FIV/ICSI outcomes (characteristics of ovarian stimulation and biological proceeds) were presented in Table 2.

Table 1. Clinical and hormonal characteristics of the SOPK group versus the Control group.

\begin{tabular}{cccc}
\hline & SOPK Group & Control Group & p Value \\
\hline Number of patients & 61 & 91 & 0.44 \\
Age of patients (years) & $32.12 \pm 3.92$ & $31.45 \pm 3.81$ & $\mathrm{p}<0.05(\mathrm{p}<0.003)$ \\
SHBG (n M/ml) & $29.58 \pm 26.59$ & $77.1 \pm 84.81$ & $\mathrm{p}<0.05(\mathrm{p}=0.0002)$ \\
Total Testosterone $(\mathrm{ng} / \mathrm{ml})$ & $0.93 \pm 0.42$ & $0.61 \pm 0.25$ & $\mathrm{p}<0.05(\mathrm{p}<0.0001)$ \\
$\Delta-4$ androstenedione $(\mathrm{ng} / \mathrm{ml})$ & $3.74 \pm 2.09$ & $1.82 \pm 0.86$ & $\mathrm{p}=0.91$ \\
FAI $(\%)$ & $16 \pm 37$ & $17 \pm 39$ & $\mathrm{p}=0.53$ \\
\hline
\end{tabular}

Data shown as mean value \pm standard deviation.

Table 2. Characteristics of FIV/ICSI outcomes in SOPK group versus Control group.

\begin{tabular}{|c|c|c|c|}
\hline & SOPK Group $(n=61)$ & Control Group $(n=91)$ & p Value \\
\hline E2 level at HCG day (pg/ml) & $3079.4 \pm 1257.6$ & $1450 \pm 845$ & $\mathrm{p}<0.05(\mathrm{p}<0.001)$ \\
\hline Duration of stimulation (days) & $11.33 \pm 2.62$ & $9.5 \pm 2.5$ & $\mathrm{p}<0.05(\mathrm{p}=0.0003)$ \\
\hline Number of ampoules of FSH administrated & $24.37 \pm 11.99$ & $24.71 \pm 10.19$ & $\mathrm{p}=0.89$ \\
\hline $\begin{array}{l}\text { Number of follicules } \\
\text { on ultasound on the ponction's day }\end{array}$ & $11.31 \pm 4.09$ & $5.09 \pm 2.34$ & $\mathrm{p}<0.05(\mathrm{p}<0.0001)$ \\
\hline Number of oocytes ponctioned & $8.40 \pm 3.83$ & $6.60 \pm 4$ & $\mathrm{p}=0.015$ \\
\hline Oocytes quality (\%) & $0.40 \pm 0.27$ & $0.48 \pm 0.25$ & $\mathrm{p}=0.20$ \\
\hline Number of embryos obtained & $3.93 \pm 3.10$ & $4.03 \pm 3.43$ & $\mathrm{p}=0.89$ \\
\hline Number of embryos transfered & $2.34 \pm 0.98$ & $2.03 \pm 0.98$ & $\mathrm{p}=0.23$ \\
\hline Embryos quality (\%) & $0.57 \pm 0.39$ & $0.39 \pm 0.31$ & $\mathrm{p}=0.05$ \\
\hline Fertilization rate $(\%)$ & $0.50 \pm 0.29$ & $0.67 \pm 0.33$ & $\mathrm{p}=0.02$ \\
\hline Cleavage rate $(\%)$ & $90.4 \pm 24.32$ & $29 \pm 44$ & $\mathrm{p}<0.05(\mathrm{p}<0.0001)$ \\
\hline
\end{tabular}

Values are given as mean number \pm standard deviation. 


\subsubsection{Characteristics of Ovarian Stimulation Phase}

The duration of stimulation, expressed by days, was significantly longer in the PCOS group comparably to the control group. (11.33 \pm 2.62 versus $9.5 \pm 2.5)$. We noted no significantly differences between the numbers of FSH's ampoules used for PCOS patients compared with controls (24.37 \pm 11.99 versus $24.71 \pm 10.19, \mathrm{p}=0.89)$. The E2 levels ( $\mathrm{pg} / \mathrm{ml})$ on the HCG administration day were significantly higher in the PCOS group comparably to the control group $(3079.4 \pm 1257.6$ versus $1450 \pm 845)$. Significantly more follicles were observed on the ultrasound on the day of puncture and more punctured oocytes were obtained in PCOS patients compared with controls, respectively $(11.31 \pm 4.09$ versus $5.09 \pm 2.34)$, and $(8.40 \pm 3.83$ versus $6.6 \pm 4)$.

\subsubsection{Characteristics of Biological Proceeds' Outcomes}

We found no significantly differences concerning oocytes quality and number of embryos obtained after applying the FIV/ICSI proceeds between the SOPK group and the control group, respectively $(0.40 \pm 0.27$ versus $0.48 \pm 0.25, p=0.20)$ and $(3.93 \pm 3.10$ versus $4.03 \pm 3.43, p=0.89)$. The embryos quality was significantly better in the SOPK, such as fertilization rate and cleavage rate, respectively $(0.57 \% \pm 0.39 \%$ versus $0.39 \% \pm$ $0.31 \%),(50 \% \pm 29 \%$ versus $67 \% \pm 33 \%)$ and $(90.4 \% \pm 24.32 \%$ versus $29 \% \pm 44 \%)$.

Table 3 showed the correlation of SHBG and IVF outcomes in SOPK group and Control group. According to our results, except a significant correlation between SHBG and number of embryos obtained in control group but not verified in SOPK group, it seemed that no other significant correlation between SHBG and FIV/ICSI outcomes was found either in SOPK or in Control group.

\section{Discussions}

The results of our study indicate that serum SHBG levels are not correlated with IVF outcomes either in SOPK group or in Control group.

The composition of follicular fluid, and secondarily blood serum, are considered as useful indicators of secretary activities and metabolism of follicular fluid cells [8] [9]. Besides, they reflect the stages of follicle maturation [10]. Sex steroids are thought to act as local regulators of granulosa cell replication, differentiation and oocyte maturation. In consequence, steroids' levels appreciation is important in determining the response to gonadotropins and the fate of individual follicles [1] [8].

In IVF proceeds, predicting ovarian response before undertaking expansive treatments is crucial. In spite of normal rates of screening parameters such as age, antral follicle count (AFC), levels of serum inhibin $\mathrm{B}$, serum anti-Müllerian hormone (AMH), basal serum follicle stimulating hormone (FSH) and estradiol (E2), basal FSH/ LH (luteinizing hormone) ratio), a large amount of infertile women respond poorly to gonadotrophins [9].

In consequence, during these last decades, different components of follicular fluid were used as indicators of maturation and quality of the follicle in the purpose to predict fertilization outcomes, such as SHBG [11].

Table 3. Comparison of SHBG-FIV/ICSI outcomes' correlation between SOPK group and Control group.

\begin{tabular}{ccccc} 
& \multicolumn{5}{c}{ Correlation SHBG-FIV/ICSI outcomes } \\
\cline { 2 - 5 } & \multicolumn{2}{c}{ SOPK Group ( $\mathrm{n}=61)$} & \multicolumn{2}{c}{ Control Group $(\mathrm{n}=91)$} \\
\cline { 2 - 5 } & $\mathrm{r}^{2}$ & $\mathrm{p}$ & $\mathrm{r}^{2}$ & $\mathrm{p}$ \\
\hline Duration of stimulation (days) & 0.005 & 0.48 & 0.034 & 0.17 \\
Number of ampoules of FSH administrated & 2.27 & 0.90 & 2.29 & 0.93 \\
Number of oocytes ponctioned & 0.027 & 0.27 & 0.099 & 0.08 \\
Oocytes quality (\%) & 0.012 & 0.31 & 0.168 & 0.02 \\
Number of embryos obtained & 2.81 & 0.94 & 0.041 & 0.096 \\
Embryos quality (\%) & 0.004 & 0.68 & 0.069 & 0.16 \\
Fertilization rate (\%) & 0.001 & 0.82 & 0.021 & 0.44 \\
Cleavage rate (\%) & 0.005 & 0.64 & 0.09 \\
\hline
\end{tabular}


SHBG, produced by granulosa lutein cells, has been found to be present in various follicular components throughout the different stages of folliculogenesis [1] [4] [12]. But, the presence of SHBG in the follicular fluid of human antral follicles was considered to be derived from the blood serum, as its concentration in both fluids was quite similar [3].

As in the blood plasma, follicular fluid steroids interact with different binding proteins, specially the SHBG which binds with high affinity intrafollicular, androgens and estrogens [3] [5] [13]. This fact highlights the importance of SHBG as a local endogenous regulator of bioavailability of steroids inside the ovary [1] [4] [5]. Besides, an increase of SHBG levels in serum and follicular fluid is observed during ovarian stimulation for IVF, presumably to regulate the bioactivity of sex steroids [12]. Consequently, a growing body of evidence has accumulated regarding regulation of ovarian function by SHBG and ovarian response to standard gonadotrophins stimulation [5]. But, conflicting results exist concerning its ability to affect fertilization rates and pregnancy outcomes [4] [7].

The Polycystic Ovary Syndrome (PCOS) is one of the most common endocrine disorders occurring in women of reproductive age that range from $6.5 \%$ to $8 \%$ using biochemical and/or clinical evidences [4] [5]. Furthermore, in women attending infertility clinics, PCOS accounts for nearly $73 \%$ of patients suffering from anovulatory infertility [4].

The relationship between PCOS and oocyte developmental competence remains obscure and incompletely understood. Considering androgen excess as noxious to follicular development, classic PCOS patients who manifest the greatest degrees of hyperandrogenism are most likely to have an abnormal intrafollicular environment and impaired developmental competence of the oocyte [14]. And this fact seems to be responsible of follicular growth abnormalities, anovulation and infertility [10]. Accordingly, after less invasive methods have failed, IVF and ICSI are considered as ideal treatment options aimed at restoring fertility for SOPK infertile women [4] [15].

As expected, our SOPK group was characterized by a significant high and perturbing androgynous status and a significantly less SHBG levels compared with Control group [16] [17]. Concerning IVF'S response profile, the SOPK group demonstrated a significantly better ovarian response after gonadotrophins administration (higher E2/HCG, shorter stimulation period, more follicles obtained and punctured) than the control group [16] [18]. After achieving the biological proceeds, the outcomes of FIV/ICSI were significantly better in the PCOS group concerning the embryo quality and the cleavage's rate [10] [15] [18].

But, it is interesting to signal that no difference was observed between the two groups after correlating these previous parameters with SHBG.

It has been demonstrated that elevated follicular fluid androgen levels were associated with lower quality oocytes and lower cleavage rates [1] [19]. The beneficial SHBG's effect on folliculogenesis is probably due to the high capacity of SHBG on binding testosterone, decreasing and inactivating androgen [1] [8] [19]. At this point it should be noted that recent clinical reports demonstrated that androgens, primarily testosterone and androstenedione, are noteworthy to enhance recruitment, promote growth and development of follicular, by promoting both theca and granulosa cell proliferation and inhibiting apoptosis [1] [19]. Theses androgen's effects concern specially the early gonadotrophin-independent stages of folliculogenesis [1] [9] [19]. Consequently, the presence of androgens at the adequate levels could increase both quantity and quality of oocytes and embryos, and improve pregnancy outcomes [1] [9].

It is worth noticing that most of the literature has been based on SHBG direct or indirect (paracrine/autocrine) effects on follicle [3] [14] from either natural or controlled ovarian stimulation cycles [8] [9] without taking into account the effect of metabolic disorders. Our choice in recruiting a group of SOPK infertile women was calculating: this syndrome illustrates the complex relationship between reproduction axis and others, specially the metabolic one [20]. Alterations in several different metabolic pathways have been implicated in the physiopathology of PCOS, including infertility, dysovulation, anovulation, abnormalities in steroid hormone regulation and insulin signaling pathways [21]-[23].

The majority of SHBG is produced by the liver, and hepatic production of SHBG is regulated by several metabolic factors including insulin and androgens [21]. SHBG may be considered as the link between metabolic and reproductive pathways, and consequently, alterations in hepatic production of SHBG can be responsible of infertility abnormalities in affected women [22].

Finally, our results show that SHBG can't be considered as an efficient predictor of FIV/ICSI outcomes in polycystic ovary syndrome. This finding could be attributed to the fact that SHBG's production is depending on 
many parameters witch differ from a patient to another, such as metabolic profile [20] [24]. Additional studies will be required to understand SHBG functions in balancing of levels of sex steroid within the follicle and under which condition metabolic disorders could interfere with this previous action during ovulation induction. And this will be the subject of our next work.

\section{Conflict of Interest}

Authors have no conflicts of interest or any financial support to declare.

\section{References}

[1] Hatsi, E., Bouba, I., Galidi, A., lazaros, L., Xita, N., Sakaloglou, P., et al. (2011) Association of Serum Association of Serum and Follicular Fluid SHBG Levels and SHBG (TAAAA)n Polymorphism with Follicle Size in Women Undergoing Ovarian Stimulation. Gynecological Endocrinology, 27, 27-32. http://dx.doi.org/10.3109/09513590.2010.493961

[2] Stener-Victorin, E., Holm, G., Labrie, F., Nilsson, L., Janson, P. and Ohlsson, C. (2010) Are There Any Sensitive and Specific Sex Steroid Markers for Polycystic Ovary Syndrome? Journal of Clinical Endocrinology \& Metabolism, 95, 810-819. http://dx.doi.org/10.1210/jc.2009-1908

[3] Forges, T., Gérard, A., Hess, K., Monnier-Barbarino, P. and Gérard, H. (2004) Expression of Sex Hormone-Binding Globulin (SHBG) in Human Granulosa-Lutein Cells. Molecular and Cellular Endocrinology, 219, 61-68. http://dx.doi.org/10.1016/j.mce.2004.01.011

[4] Forges, T., Gérard, A., Monnier-Barbarino, P. and Gérard, H. (2005) Sex Hormone-Binding Globulin (SHBG) in the Ovary. Treballs de la $S C B, \mathbf{5 6}, 117-125$.

[5] Lazaros, L.A., Hatzi, E.G., Pamporaki, C.E., Sakaloglou, P.I., Xita, N.V., Markoula, S.I., et al. (2012) The Ovarian Response to Standard Gonadotrophin Stimulation Depends on FSHR, SHBG and CYP19 Gene Synergism. Journal of Assisted Reproduction and Genetics, 29, 1185-1191. http://dx.doi.org/10.1007/s10815-012-9849-0

[6] De Leo, V., Di Sabatino, A., Musacchio, M.C., Morgante, G., Scolaro, V., Cianci, A., et al. (2010) Effect of Oral Contraceptives on Markers of Hyperandrogenism and SHBG in Women with Polycystic Ovary Syndrome. Contraception, 82, 276-280. http://dx.doi.org/10.1016/j.contraception.2010.04.002

[7] Lin, K.C. and Sun, M.J. (2005) Relationship between Sex Hormone-Binding Globulin and Pregnancy Outcome in Women Undergoing Controlled Ovarian Hyperstimulation for Assisted Reproduction. Endocrine Journal, 52, 407-412. http://dx.doi.org/10.1507/endocrj.52.407

[8] Hogeveen, K.N., Cousin, P., Pugeat, M., Dewailly, D., Soudan, B. and Hammond, G.L. (2002) Human Sex HormoneBinding Globulin Variants Associated with Hyperandrogenism and Ovarian Dysfunction. Journal of Clinical Investigation, 109, 973-981. http://dx.doi.org/10.1172/JCI0214060

[9] Qin, Y., Zhao, Z., Sun, M., Geng, L., Che, L. and Chen, Z.J. (2011) Association of Basal Serum Testosterone Levels with Ovarian Response and in Vitro Fertilization Outcome. Reproductive Biology and Endocrinology, 9, 9. http://dx.doi.org/10.1186/1477-7827-9-9

[10] Dumesic, D.A., Padmanabhan, V. and Abbott, D.H. (2008) Polycystic Ovary Syndrome and Oocyte Developmental Competence. Obstetrical \& Gynecological Survey, 63, 39-48. http://dx.doi.org/10.1097/OGX.0b013e31815e85fc

[11] Zhang, H., Legro, R.S., Zhang, J., Zhang, L., Chen, X., Huang, H., et al. (2010) Decision Trees for Identifying Predictors of Treatment Effectiveness in Clinical Trials and Its Application to Ovulation in a Study of Women with Polycystic Ovary Syndrome. Human Reproduction, 25, 2612-2621. http://dx.doi.org/10.1093/humrep/deq210

[12] Kahn, S.M., Hryb, D.J., Nakhla, A.M., Romas, N.A. and Rosner, W. (2002) Sex Hormone-Binding Globulin Is Synthesized in Target Cells. Journal of Endocrinology, 175, 113-120. http://dx.doi.org/10.1677/joe.0.1750113

[13] Xita, N., Georgiou, I., Lazaros, L., Psofaki, V., Kolios, G. and Tsatsoulis, A. (2008) The Synergistic Effect of Sex Hormone-Binding Globulin and aroMatase Genes on Polycystic Ovary Syndrome Phenotype. European Journal of Endocrinology, 158, 861-865. http://dx.doi.org/10.1530/EJE-07-0905

[14] Rosner, W., Hryb, D.J., Kahn, S.M., Nakhla, A.M. and Romas, N.A. (2010) Interactions of Sex Hormone-Binding Globulin with Target Cells. Molecular and Cellular Endocrinology, 316, 79-85. http://dx.doi.org/10.1016/j.mce.2009.08.009

[15] Ludwig, M., Finas, D., Al-Hasani, S., Diedrich, K. and Ortmann, O. (1999) Oocyte Quality and Treatment Outcome in Intracytoplasmic Sperm Injection Cycles of Polycystic Ovarian Syndrome Patients. Human Reproduction, 14, $354-358$. http://dx.doi.org/10.1093/humrep/14.2.354

[16] Heijnen, E.M.E.W., Eijkemans, M.J.C., Hughes, E.G., Laven, J.S.E., Macklon, N.S. and Fauser, B.C.J.M. (2006) A Meta-Analysis of Outcomes of Conventional IVF in Women with Polycystic Ovary Syndrome. Human Reproduction, 
12, 13-21. http://dx.doi.org/10.1093/humupd/dmi036

[17] Zhong, Y.P., Ying, Y., Wu, H.T., Zhou, C.Q., Xu, Y.W., Wang, Q., et al. (2012) Comparison of Endocrine Profile and in Vitro Fertilization Outcome in Patients with PCOS, Ovulatory PCO, or Normal Ovaries. International Journal of Endocrinology, 2012, Article ID: 492803, 6 p.

[18] Beydoun, H.A., Stadtmauer, L., Beydoun, M.A., Russell, H., Zhao, Y. and Oehninger, S. (2009) Polycystic Ovary Syndrome, Body Mass Index and Outcomes of Assisted Reproductive Technologies. Reproductive Biomedicine Online, 18, 856-863. http://dx.doi.org/10.1016/S1472-6483(10)60037-5

[19] Gleicher, N., Weghofer, A. and Barad, D.H. (2011) The Role of Androgens in Follicle Maturation and Ovulation Induction: Friend or Foe of Infertility Treatment? Reproductive Biology and Endocrinology, 9, 116. http://dx.doi.org/10.1186/1477-7827-9-116

[20] Apridonidze, T., Essah, P.A., Iuorno, M.J. and Nestler, J.E. (2005) Prevalence and Characteristics of the Metabolic Syndrome in Women with Polycystic Ovary Syndrome. The Journal of Clinical Endocrinology and Metabolism, 90, 1929-1935. http://dx.doi.org/10.1210/jc.2004-1045

[21] Wickham III, E.P., Ewens, K.G., Legro, R.S., Dunaif, A., Nestler, J.E. and Strauss III, J.F. (2011) Polymorphisms in the SHBG Gene Influence Serum SHBG Levels in Women with Polycystic Ovary Syndrome. The Journal of Clinical Endocrinology and Metabolism, 96, E719-E727. http://dx.doi.org/10.1210/jc.2010-1842

[22] Ola, S.I. and Sun, Q.Y. (2012) Factors Influencing the Biochemical Markers for Predicting Mammalian Oocyte Quality. Journal of Reproduction and Development, 58, 385-392. http://dx.doi.org/10.1262/jrd.11-084H

[23] Wildman, R.P., Wang, D., Fernandez, I., Mancuso, P., Santoro, N., Scherer, P.E., et al. (2012) Associations of Testosterone and Sex Hormone Binding Globulin with Adipose Tissue Hormones in Midlife Women. Obesity, 2012, 109. http://dx.doi.org/10.1038/oby.2012.109

[24] Soriguer, F., Rubio-Martın, E., Fernandez, D., Valdes, S., Garcia-Escobar, E., Martın-Nunez, G.M., et al. (2012) Testosterone, SHBG and Risk of Type 2 Diabetes in the Second Evaluation of the Pizarra Cohort study. European Journal of Clinical Investigation, 42, 79-85. http://dx.doi.org/10.1111/j.1365-2362.2011.02559.x 